\title{
RESONANCE RAMAN INVESTIGATIONS OF LOW ENERGETIC MODES IN THE ORGANIC SUPERCONDUCTORS $\alpha_{\mathrm{t}}-(\mathrm{BEDT}-\mathrm{TTF})_{2} \mathrm{I}_{3}$ AND $\beta$-(BEDT-TTF $)_{2} \mathrm{IAuI}$
}

\author{
T. Ludwig, D. Schweitzer
}

3. Physikalisches Institut, Universität Stuttgart

Pfaffenwaldring 57, 70550 Stuttgart, Germany

AND H.J. KELLER

Anorganisch-Chemisches Institut, Universität Heidelberg

Im Neuenheimer Feld 270, 69120 Heidelberg, Germany

A study of low energetic modes in the resonance Raman spectra of the organic superconductors $\alpha_{\mathrm{t}}$-(BEDT-TTF) $)_{2} \mathrm{I}_{3}\left(T_{c}=8 \mathrm{~K}\right)$ and $\beta$-(BEDT$-\mathrm{TTF})_{2} \mathrm{IAuI}\left(T_{\mathrm{c}}=4 \mathrm{~K}\right)$ in the temperature range of $1.5-100 \mathrm{~K}$ was performed. In both materials, a strong vanishing of low energetic phonon bands (at $30 \mathrm{~cm}^{-1}$ and $40 \mathrm{~cm}^{-1}$ for $\alpha_{\mathrm{t}}-(\mathrm{BEDT}-\mathrm{TTF})_{2} \mathrm{I}_{3}$ and at $27 \mathrm{~cm}^{-1}$ and $33 \mathrm{~cm}^{-1}$ for $\beta$-(BEDT-TTF $\left.)_{2} I A u I\right)$ below $T_{c}$ was observed. For the symmetric stretching mode of the $\mathrm{I}_{3}^{-}$and the $\mathrm{IAuI}^{-}$anion at $120 \mathrm{~cm}^{-1}$ no change below $T_{\mathrm{c}}$ was found. In addition, wavelength dependent measurements on $\alpha$-(BEDT-TTF) $)_{2} \mathrm{I}_{3}$ were performed, indicating that the modes around $30 \mathrm{~cm}^{-1}$ are probably librational modes of the BEDT-TTF molecules. PACS numbers: $74.70 . K n, 78.30 .-\mathbf{j}$

\section{Introduction}

The organic superconductors with the highest transition temperatures are all radical salts of the donor bis(ethylene-dithio)tetrathiafulvalene (BEDT-TTF). Raman spectroscopy is a useful tool to understand the nature of the superconducting mechanism in these compounds, because it is based on the electron-phonon interaction and therefore is able to probe sensitively both the phonon and the electronic subsystems and their coupling. In conventional [1] and high- $T_{\mathrm{c}}$ superconductors (e.g. [2]) Raman spectroscopy is often used to determine the superconducting gap through the change in the phonon structure on going from the normal to the superconducting state due to the interaction of the phonons and the superconducting gap. 
Recently, in Raman spectra of $\alpha_{t^{-}}$and $\beta$-(BEDT-TTF $)_{2} \mathrm{I}_{3}$ by cooling down the crystals from the normal metallic state into the superconducting state, a weakening and shift of low frequency optical phonons around $30 \mathrm{~cm}^{-1}$ as well as a strong decrease in the background was observed [3, 4]. As an explanation, in Refs. [3] and [4], a phonon-superconducting amplitude mode interaction in terms of the Balseiro-Falicov model [5] was proposed. In order to clarify several open questions, more extended measurements were made on $\alpha_{\mathrm{t}}$-(BEDT-TTF $)_{2} \mathrm{I}_{3}$ as well as on another organic superconductor, $\beta$-(BEDT-TTF $)_{2}$ IAuI.

\section{Experimental}

Raman measurements on single crystals of $\alpha_{\mathrm{t}}$-(BEDT-TTF $)_{2} \mathrm{I}_{3}$ and $\beta$-(BEDT-TTF $)_{2}$ IAuI were made in a $90^{\circ}$ scattering configuration using several lines of an argon-ion laser and a krypton-ion laser. In contrast to [3, 4], in the measurements described here, the angle between the crystal surface and the scattered radiation was only about $20^{\circ}$, while in Refs. [3] and [4] this angle was $45^{\circ}$. The measurements at $1.5 \mathrm{~K}$ were performed in a liquid helium bath cryostat, all others in a continuous flow-cryostat. The laser beam was filtered with a monochromator and an additional interference filter to suppress plasma lines. A Spex 1404 double monochromator with a space filter was used to disperse the scattered radiation. The power of the laser beam was kept below $35 \mathrm{~mW}$ and not completely focused in order to prevent sample damages and laser heating. The $\alpha_{\mathrm{t}}$ (BEDT-TTF $)_{2} \mathrm{I}_{3}$ crystals were prepared from $\alpha$-(BEDT-TTF $)_{2} \mathrm{I}_{3}$ crystals by tempering for $16 \mathrm{~h}$ at $95^{\circ} \mathrm{C}$. The intensity of the symmetric stretching mode of the $\mathrm{I}_{3}^{-}$and the $\mathrm{IAuI}^{-}$ anion at $120 \mathrm{~cm}^{-1}$ was maintained at a constant. level during the temperature measurements in order to normalise the spectra.

\section{Results and discussion}

Figure 1 shows the low energetic part of the Raman spectrum of $\alpha_{\mathrm{t}}$-(BEDT$-\mathrm{TTF})_{2} \mathrm{I}_{3}$ above and below $T_{\mathrm{c}}=8 \mathrm{~K}$ similar to the observations of $[3,4]$.

In our results here, on going from the normal conducting phase to the superconducting phase, we found a similar strong decrease in the intensity of the phonon bands at $30 \mathrm{~cm}^{-1}$ and $40 \mathrm{~cm}^{-1}$, but only a weak decrease in the intensity of the background. In the normal conducting phase between $10 \mathrm{~K}$ and $100 \mathrm{~K}$, no further change is observed.

The fact that the decrease in the intensity of the background in these measurements here is weaker might be due to the transformation of the crystals from the $\alpha$ - to the $\alpha_{\mathrm{t}}$-phase by tempering or, more likely, due to the different angle between the crystal surface and the scattered radiation.

In order to prove whether this decrease is real or not, additional measurements on $\beta$-(BEDT-TTF) ${ }_{2} \mathrm{IAuI}$ were performed.

The low energetic part of the Raman spectrum of superconducting single crystals of $\beta$-(BEDT-TTF) ${ }_{2} \mathrm{IAuI}$ at $T=1.5 \mathrm{~K}$ is shown in Fig. 2 . This spectrum is similar to those reported by Swietlik et al. [6].

It consists mainly of the symmetric stretching mode of the $\mathrm{IAuI}^{-}$anion at $120 \mathrm{~cm}^{-1}$ and very weak bands around $40 \mathrm{~cm}^{-1}$ and $100 \mathrm{~cm}^{-1}$. 


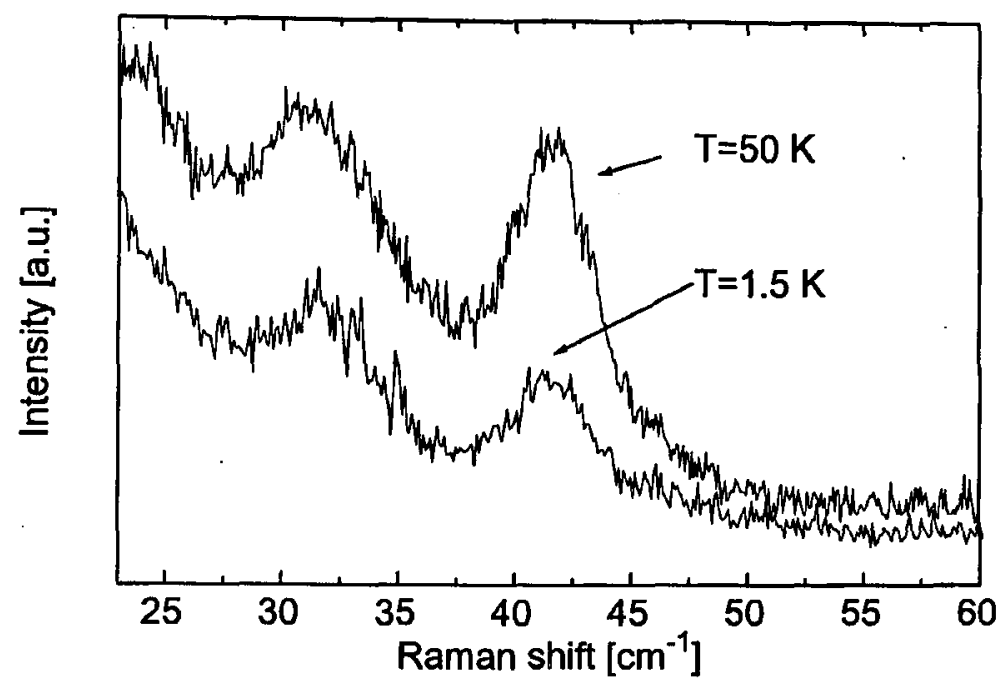

Fig. 1. Low energetic part of the Raman spectrum of $\alpha_{t}$-(BEDT-TTF) $)_{2} I_{3}$ above and below $T_{\mathrm{c}}=8 \mathrm{~K}$ (excitation $\lambda=457.9 \mathrm{~nm}$ ).

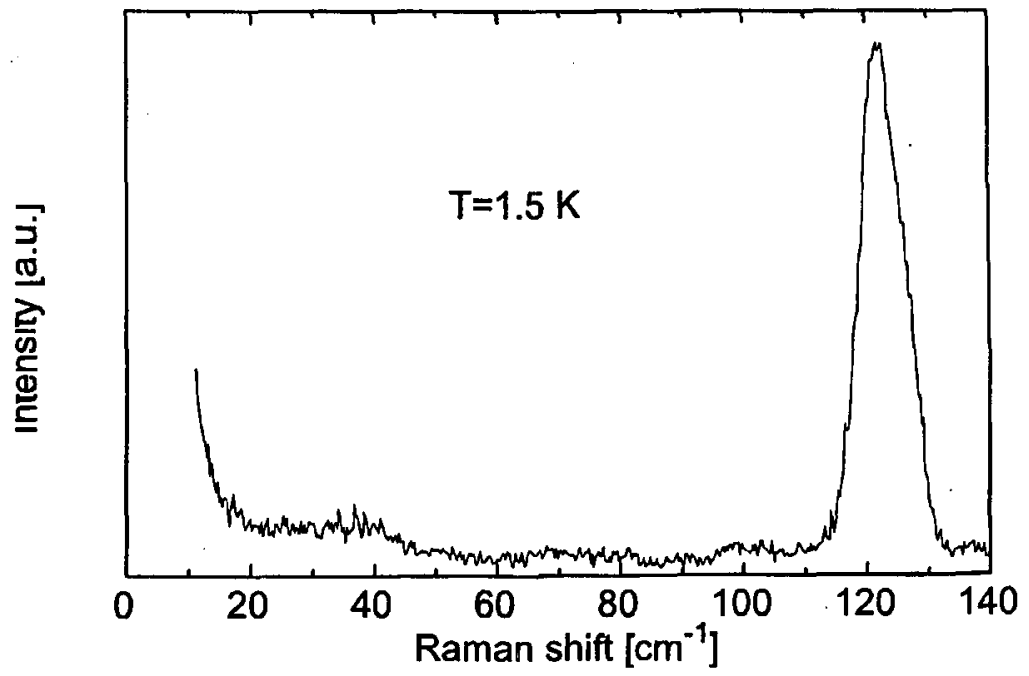

Fig. 2. Low energetic part of the Raman spectrum of superconducting $\beta$-(BEDT$-\mathrm{TTF})_{2} \mathrm{IAuI}$ single crystals (excitation $\lambda=457.9 \mathrm{~nm}$ ).

However, in the normal conducting phase at $T>T_{\mathrm{c}}=4 \mathrm{~K}$ (see Fig. 3 at $50 \mathrm{~K}$ ) in the best resolved spectra two lines at $27 \mathrm{~cm}^{-1}$ and $33 \mathrm{~cm}^{-1}$ are observed, while in the superconducting phase at $T<T_{\mathrm{c}}=4 \mathrm{~K}$ (Fig. 3 , middle at $1.5 \mathrm{~K}$ ), a strong weakening of these bands can be seen and a small suppression of the background occurs (Fig. 3, bottom). The very weak structure around $48 \mathrm{~cm}^{-1}$ is probably due to a plasma line of the argon-ion laser, working at $457.9 \mathrm{~nm}$. 


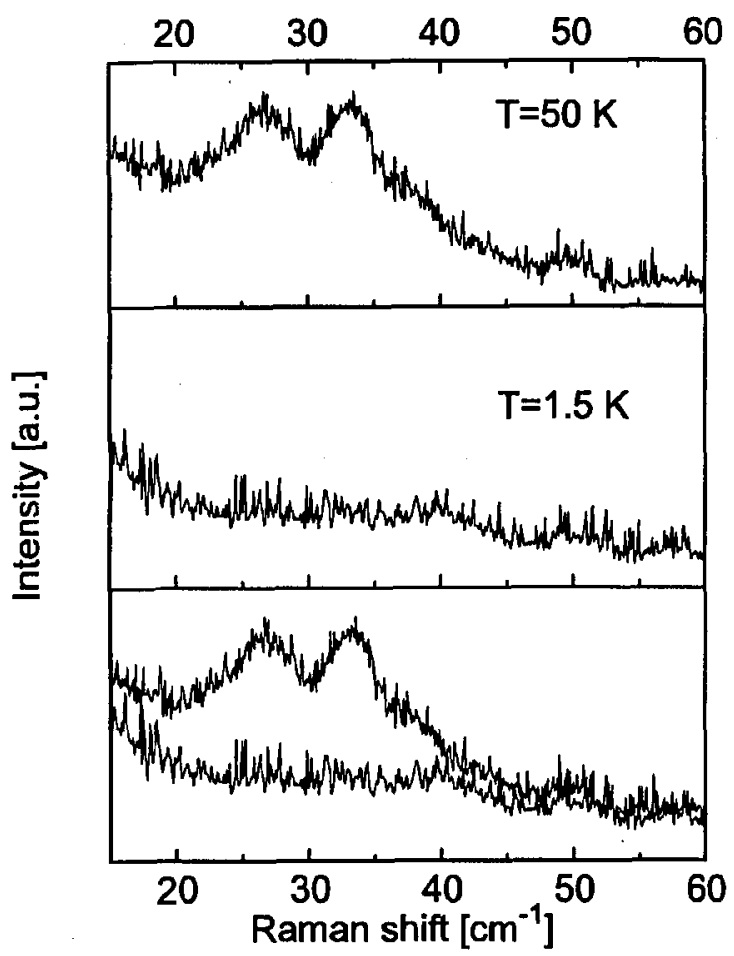

Fig. 3. Low energetic part of the Raman spectra of $\beta$-(BEDT-TTF) ${ }_{2}$ IAuI single crystals above $T_{\mathrm{c}}$ (top), below $T_{\mathrm{c}}$ (middle) as well as both together (bottom) (excitation $\lambda=$ $457.9 \mathrm{~nm}$ ).

No change occurs in the Raman spectra of the normal phase between $10 \mathrm{~K}$ and $50 \mathrm{~K}$ and for the symmetric stretching mode of the $\mathrm{IAuI}^{-}$anion at $120 \mathrm{~cm}^{-1}$ in the whole temperature range between $1.5 \mathrm{~K}$ and $50 \mathrm{~K}$.

According to tunnelling data of Hawley et al. [7], the superconducting gap is in the same energy range as the vanishing bands while in the tunnelling data of Nowack et al. [8] a much smaller value for the gap was reported. The reason for this difference might be due to a strong anisotropy of the gap in $\beta$-(BEDT-TTF $)_{2}$ IAuI [8]. Therefore, as in $\alpha_{\mathrm{t}}$-(BEDT-TTF $)_{2} \mathrm{I}_{3}$, an interaction between the gap and the low energetic phonons around $30 \mathrm{~cm}^{-1}$ is probably the reason for the change in the phonon structure below $T_{\mathrm{c}}$.

To clarify the origin of the modes around $30 \mathrm{~cm}^{-1}$, we performed additional measurements with different excitation wavelengths on the semiconducting $\alpha$-(BEDT-TTF $)_{2} \mathrm{I}_{3}$ phase at $T=20 \mathrm{~K}$.

The Raman scattering of the symmetric stretching mode of the $\mathrm{I}_{3}^{-}$anion has a resonant character with its maximum at an excitation at $488.0 \mathrm{~nm}$. For a fixed excitation wavelength, no temperature-dependent change in the spectra occurs. However, the relative intensity of the band at $30 \mathrm{~cm}^{-1}$ compared to the band at 


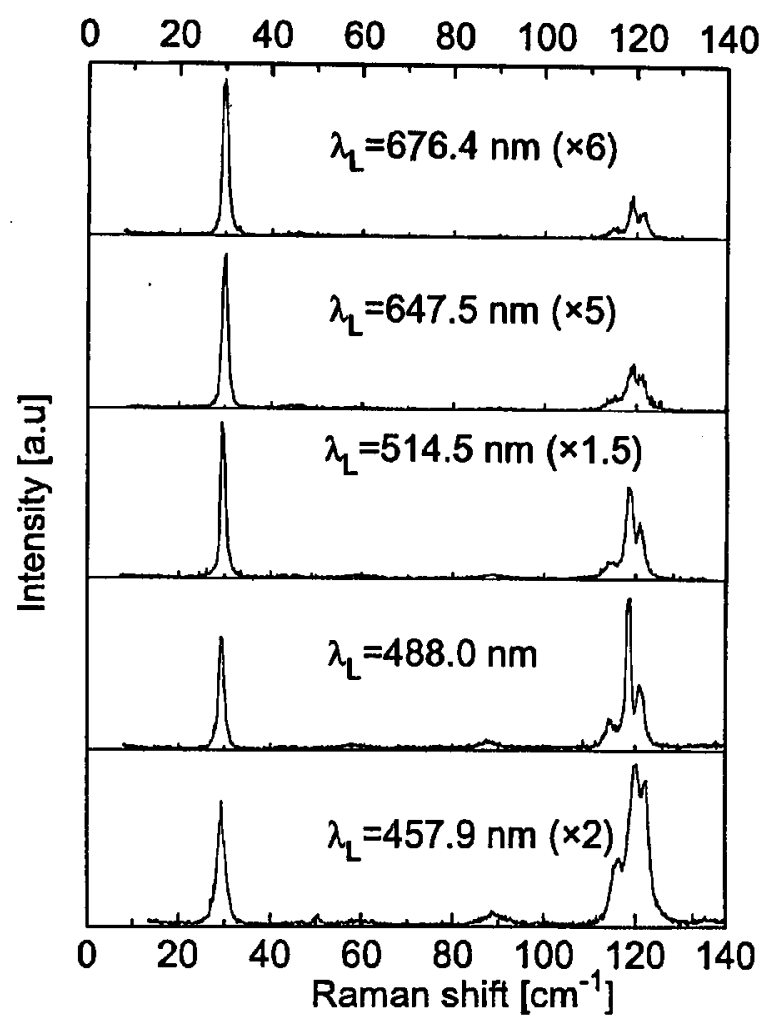

Fig. 4. Low energetic part of the Raman spectrum of $\alpha$-(BEDT-TTF) $)_{2} \mathrm{I}_{3}$ at $T=20 \mathrm{~K}$ for different excitation lines, all measurements except the spectrum for $\lambda=488.0 \mathrm{~nm}$ are scaled as indicated.

$120 \mathrm{~cm}^{-1}$ undergoes a drastic increase on going to longer excitation wavelengths for a fixed temperature (Fig. 4). In our opinion, this indicates that the mode at $30 \mathrm{~cm}^{-1}$ is not a librational mode of the $\mathrm{I}_{3}^{-}$anion but maybe an external mode of (BEDT-TTF)- $\mathrm{I}_{3}$, also seen in IR measurements [9] or a librational mode of the BEDT-TTF molecules, as pointed out in [3]. Due to the fact that the spectral region of the vanishing bands is close to this mode, the origin of the low lying bands in both superconducting materials might be the same as for the mode at $30 \mathrm{~cm}^{-1}$ in $\alpha$-(BEDT-TTF $)_{2} \mathbf{I}_{3}$.

\section{Conclusions}

In our experiments, we observed a strong weakening of low frequency phonons at about $30 \mathrm{~cm}^{-1}$ and $40 \mathrm{~cm}^{-1}$ in $\alpha_{\mathrm{t}}$ (BEDT-TTF) ${ }_{2} \mathrm{I}_{3}$ and at $27 \mathrm{~cm}^{-1}$ and $33 \mathrm{~cm}^{-1}$ in $\beta$-(BEDT-TTF $)_{2} \mathrm{IAuI}$ below $T_{\mathrm{c}}$ in the superconducting state due to an interaction between some phonons and the superconducting gap. The origin of the vanishing bands might be an external mode of (BEDT-TTF)- $\mathrm{I}_{3}$ or a librational mode of BEDT-TTF. The fact that the reduction of the background scattering 
in our measurements is weaker than in Refs. $[3,4]$ might be due to the different scattering angle, used in our experiments here. Further experiments are necessary to clarify this question.

\section{References}

[1] R. Sooryakumar, M. Klein, Phys. Rev. Lett. 45, 660 (1980).

[2] E.T. Heyen, M. Cardona, J. Karpinski, E. Kaldis, S. Rusiecki, Phys. Rev. B 43, 12958 (1991).

[3] K.I. Pokhodnia, A. Graja, M. Weger, D. Schweitzer, Z. Phys. B 90, 127 (1993).

[4] A. Graja, K.I. Pokhodnia, M. Weger, D. Schweitzer, Synth. Met. 56, 2477 (1993).

[5] C.A. Balseiro, L.M. Falicov, Phys. Rev. Lett. 45, 662 (1980).

[6] R. Swietlik, H. Grimm, D. Schweitzer, Z. Nat.forsch. A 42, 603 (1987).

[7] M.E. Hawley, K.E. Gray, B.D. Terris, H.H. Wang, K.D. Carlson, J.M. Williams, Phys. Rev. Lett. 57, 629 (1986).

[8] A. Nowack, U. Poppe, M. Weger, D. Schweitzer, H. Schwenck, Z. Phys. B. 68, 41 (1987).

[9] V. Zelezny, J. Petzelt, R. Swietlik, B.P. Gorshunov, A.A. Volkov, G.V. Kozlov, D. Schweitzer, H.J. Keller, J. Phys. (France) 51, 869 (1990). 